\title{
RECHERCHES SUR LA CROISSANCE DES MAMMIFËRES. - SES RELATIONS AVEC LA COMPOSITION CHIMIQUE DU LAIT DE LA MËRE
}

\author{
par G. BELLE, \\ Docteur-Vétérinaire
}

(Suite).

\section{VEAU}

Il est assez difficile d'indiquer d'une façon précise l'âge auquel le veau est sevré. Dès sa naissance, le veau peut boire au seau et être privé de sa mère. Sa croissance se poursuivra aussi normalement et il n'aura pas à souffrir de cette substitution prématurée. Bien souvent d'ailleurs, pour des raisons éconcmiques, le veau est séparé de sa mère dès sa naissance; on l'alimente tout d'abord a vec du lait de vache pris soit au biberon, soit au seau, et dès le $3^{\mathrm{e}}$ jour, on l'alimente avec du lait écrémé additionné de farineux. Ainsi. suivant que le veau est destiné à la boucherie ou au contraire à être élevé, son alimentation varie. Dıns le premier cas, on le sépare de sa mère pour lui donner du lait et des farineux; ón le sèvre de très bonne heure. Dans le second cas, on le laisse avec sa mère et il peut téter soit à discrétion, soit à heures fixes. L'époque à laquelle le veau abandonne définitivement l'alimentation lactée est extrêmement variable; d'après CoRnevin, le sevrage se fait normalement à l'âge de deux mois, et jusqu'à ce moment, la quantité de lait absorbée est de 300 litres environ. Boussingault pense que, dès la $3^{\mathrm{e}}$ semaine, on peut donner du foin de bonne qualité ; on augmente progressivement la quantité jusqu'au jour où le lait est définitivement supprimé ; on peut, d'après cet auteur, sevrer à 1 mois et demi environ.

D'après d'autres auteurs, le sevrage n'aurait lieu qu'à 5 mois environ. Nos observations nous permettent de fixer à 4 mois, 4 mois et demi en moyenne, l'époque à laquelle on supprime définitivement le lait. Il faut remarquer que la vache est depuis fort longtem ps adaptée à une sécrétion lactée que l'on peut qualifier d'anormale, par rapport à sa quantité, parce que, développée par sélection et par hérédité, on est arrivé à faire produire à la vache beaucoup plus de lait qu'il n'est nézessaire pour l'alimentation de son petit.

Chez la jument, il n'en est pas ainsi ; cette dernière produit en général une quantité de lait juste suffisante aux besoins du poulain, et ceci établit une différence entre les espèzes bovine et équine.

Das pesées régulières faites sur plusieurs veaux nourris exclusivement avec du lait nous ont permis d'établir le graphique ( $\mathrm{Pl}$. X), le poids moyen d'un veau pendant les 40 premiers jours de sa vie.

Durant les dix premiers jours, le veau prend en moyenne quotidiennement 2 ou 3 kilogs; à partir du $10^{\mathrm{e}}$ jour, l'accroissement 
PL. X.

CROISSANCE MOYENNE JOURNALIÈRE DU VEAU

Accroissement moyen tous les 2 jours pour 2 veaux

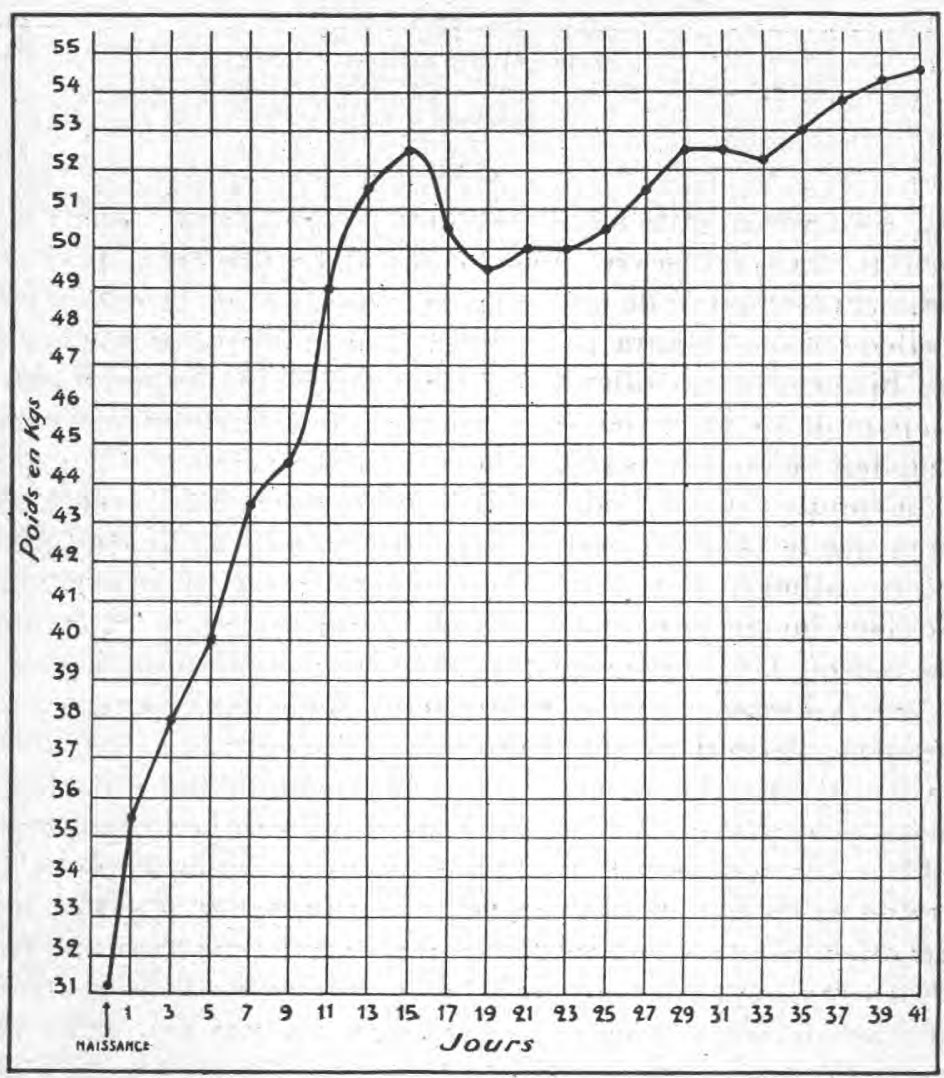

diminue. Certains animaux augmentent seulement de 1 kilog, ou $1 \mathrm{~kg} .500$; tandis que le poids de certains autres reste stationnaire ou même, quelquefois, diminue.

L'augmentation régulière reprend vers le $30^{\mathrm{e}}$ jour et se poursuit, mais est plus faible que précédemment : 1 kilog environ.

Pendant le premier mois, la croissanse pondérale est done de $20 \mathrm{~kg}$. 500 en moyenne, ce qui représente une augmentation journalière de $0 \mathrm{~kg}$. 700 . Nous a vons constaté un accroissement plus considérable sur de jeunes animaux ; l'allaitement était pratiqué d'une façon rationnelle : les veaux étaient séparés de leur mère et réunis à elle seulement aux heures de repas. $\mathrm{L}_{\mathrm{s}}$ nombre des tétées, fixé à six par jour durant la première semaine, fut progressivement diminué dans les jours suivants, afin d'arriver à trois au cours de la quatrième semaine. Le sevrage eut lieu à 3 mois. 
Une bête flamande pesait 40 kilogs à sa naissance et a pris jusqu'au sevrage (94 ${ }^{\mathrm{e}}$ jour), 95 kilogs; l'augmentation journalière était done de $1 \mathrm{~kg} .18$ environ.

Un veau ferrandais de $56 \mathrm{~kg}$. a vait augmenté de $121 \mathrm{~kg}$. au moment où il fut sevré (92 $\mathrm{e}$ jour), ce qui représente un accroissement quotidien de $1 \mathrm{~kg} .316$.

Un veau montbéliard, de $46 \mathrm{~kg}$. à la mise bas, prit en 15 jours $99 \mathrm{~kg}$., soit par jour $1 \mathrm{~kg} .164$.

Des animaux, sevrés à 2 mois et demi, ont accusé depuis la naissance une augmentation journalière de $1 \mathrm{~kg} .050$ environ. BoussinGAULT pense qu'un veau peut être sevré à 1 mois et demi environ ; il évalue à 300-400 litres la quantité de lait absorbé par un veau pen. dant un allaitement de 42 jours. Un veau a bsorbe par jour 8 à 10 litres de lait en moyenne ; immédiatement a près la naissance, le veau ne prend que 5 ou 6 litres au maximum. Progressivement, il augmente sa ration, et peut absorber quotidiennement jusqu'à 11 et 12 litres. Pour calculer la quantité exacte de lait pris à différents âges, dans une journée, Boussingault eut l'idée de peser les veaux avant et a près leur repas ; dans un repas, un veau nouveau-né prend environ $1 \mathrm{~kg}$. 500 de lait ; ̀̀ 13 jours, $3 \mathrm{~kg}$. 700 .

Ernest Perrault de Jotemps estime que, dans les sept premiers jours, le veau prend quotidiennement de $1 \mathrm{~kg}$. 160 à $1 \mathrm{~kg}$. 530 . Du $7^{\mathrm{e}}$ au $16^{\mathrm{e}}$ jour, cette augmentation pondérale est un peu plus faible que précédemment: $1 \mathrm{~kg}$. 200. Pour neuf veaux engraissés pour la boucherie, la moyenne de l'augmentation pondérale durant l'allaitement de 22 jours a été de $1 \mathrm{~kg}$. 200 par jour.

C'est pendant l'allaitement que la croissance pondérale du veau est la plus rapide. Après le sevrage, l'augmentation journalière en poids tombe progressivement à $1 \mathrm{~kg}$., $0 \mathrm{~kg}$. 860 pour a boutir à $150-200$ grammes environ, au-dessus de 3 ans.

\section{POULAIN}

C'est en général à 6 mois que le poulain est sevré ; mais, dès l'âge de 3 mois et demi, 4 mois, il commence à manger un peu de foin ou de paille et il arrive parfaitement à substituer ces aliments au tait.

L'accroissement se fait en poids et en volume ; nous envisagerons done successivement chacun de ces deux facteurs :

$1^{\circ}$ Accroissement en poids. - L'augmentation de poids variesuivant que l'on a affaire à un cheval fin ou à un cheval de gros trait. Il est donc indispensable de séparer nettement ces deux catégories a vant de donner un chiffre moyen indiquant l'augmentation de poids en un temps donné chez le poulain, en général,

Grâce à l'extrême eomplaisance de M. le Directeur des Haras de 
Pompadour, j'ai pu me documenter sur la croissance en poids des chevaux arabe et anglo-arabe. On trouvera ci-joints les graphiques des Pl. XI et XII sur l'accroissement du poids du poulain et de la pouliche arabe et anglo-arabe depuis la naissance jusqu'au sevrage, tous les 5 jours, jusqu'au $90^{\mathrm{e}}$ jour, puis tous les 10 jours jusqu'au $180^{\mathrm{e}}$ jour.

PL. XI.

\section{GROISSANCE DU POULAIN ANGLO-ARABE (mâle et femelle)}

(Croissance du poulain anglo-arabe ( 5 mâles, 5 femelles) depuis la naissance jusqu'au $60^{\circ}$ jour, tous les 10 jours)

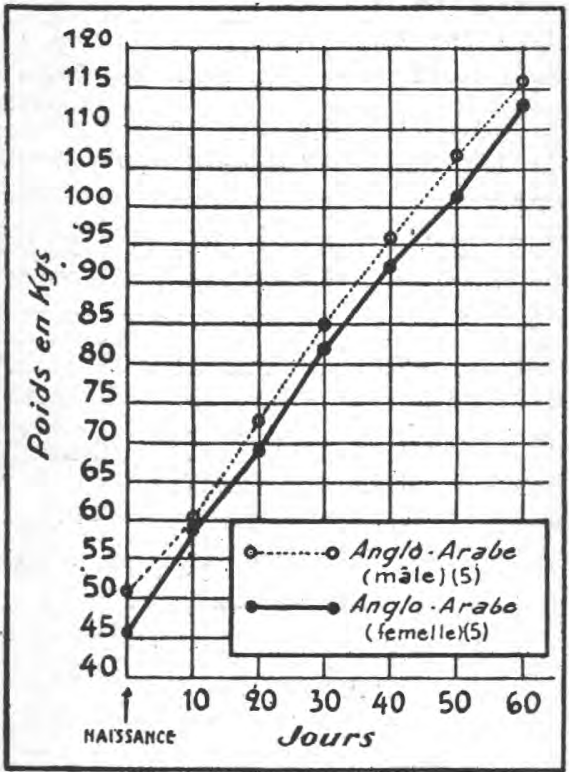

L'examen de ces chiffres nous permet de diviser en deux parties; d'une égale durée, la période d'allaitement du poulain arabe et anglo-arabe.

a) De la naissance au $60^{\mathrm{e}}$ jour, en effet, l'augmentation journalière de poids dépasse presque toujours 1 kilogramme: elle varie de $0 \mathrm{~kg} .800$ à $1 \mathrm{~kg}$. 600 ; nous trouvons exceptionnellement entre le 50e et le $55^{\mathrm{e}}$ jour une augmentation de $0 \mathrm{~kg}$. 500 seulement.

L'accroissement journalier moyen pendant les 2 premiers mois de l'existence est d'environ $1 \mathrm{~kg} .100$.

b) $D u 61^{\mathrm{e}}$ au $180^{\mathrm{e}}$ jour, l'augmentation de poids est bien moins forte ; rarement on constate une différence de $1 \mathrm{kilog}$. entre deux pesées consécutives. La croissance se ralentit beaucoup; l'accroissement varie entre $0 \mathrm{~kg}$. 550 et $0 \mathrm{~kg}$. 900 , ce qui permet d'admettre, 
comme chiffre moyen de l'augmentation journalière de poids pendant le dernier mois de l'allaitement, $0 \mathrm{~kg}$. 724.

PL. XII.

\section{CROISSANCE QUOTIDIENNE DU POULAIN DEPUIS LA NAISSANGE JUSQU'AU $180^{\circ}$ JOUR}

(Croissance du poulain tous les 5 jours jusqu'au 90e jour, puis tous les 10 jours jusqu'au $180^{\circ}$ jour. Lot de 8 poulains nés en 1925 ) (du 10 mars au 5 avril)

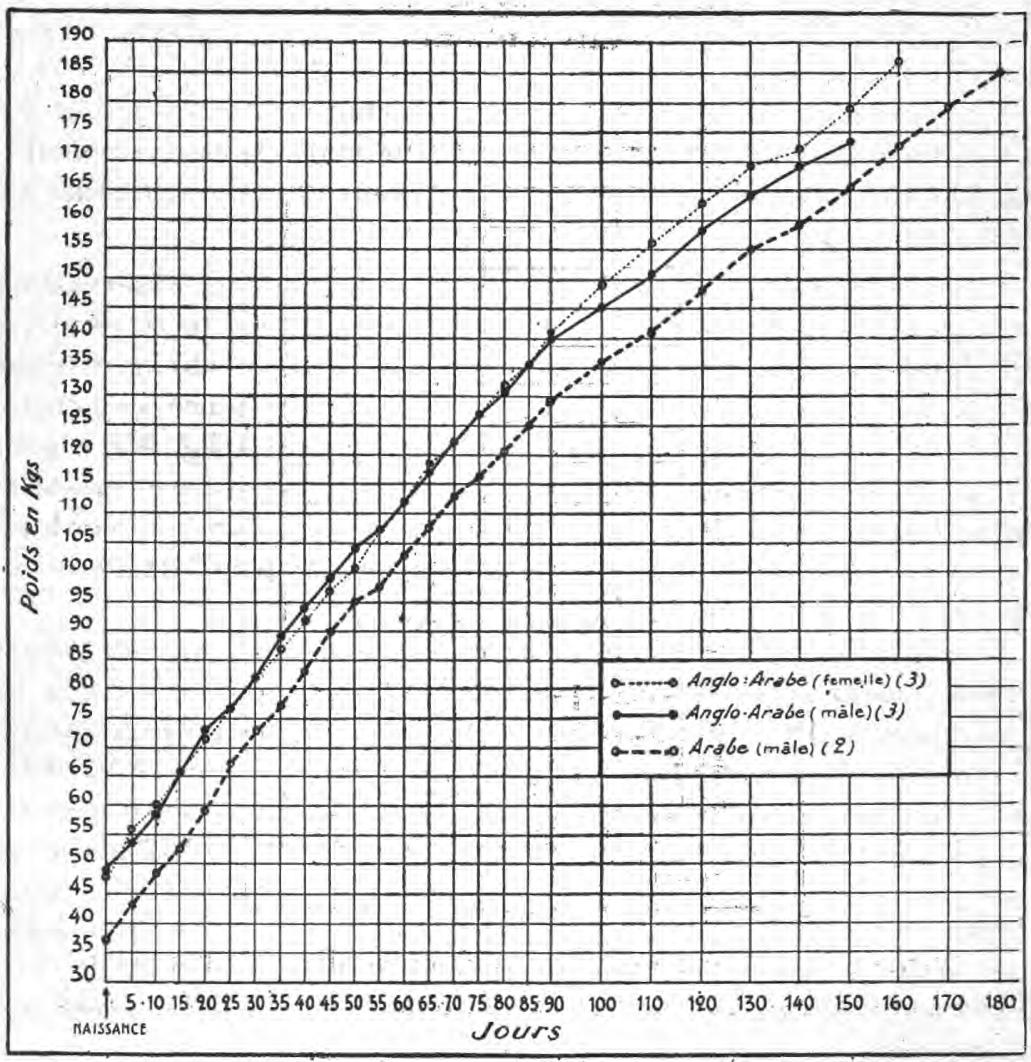

L'accroissement journalier baisse progressivement et insensible-

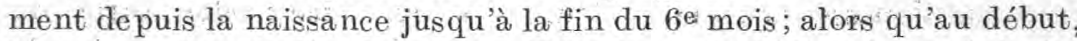
le poulain prend $1 \mathrm{~kg} .200,1 \mathrm{~kg}$. 300 par jour et davantage, à la fin du $1{ }^{\text {er }}$ mois, l'augmentation se traduit par $1 \mathrm{~kg}$. 100, et même $1 \mathrm{~kg}$; re taux d'un kilogramme se maintient jusqua $60^{\mathrm{e}}-70^{\mathrm{e}}$ jour. Dans la suite, l'augmentation journalière moyenne n'atteint pas le kilog., on la voit baisser insensiblement $\left(0 \mathrm{~kg}\right.$. $850,0 \mathrm{~kg}$. 860 vers le $90^{\mathrm{e}}-100^{\mathrm{e}}$ jour; puis $0 \mathrm{~kg}$. $700,0 \mathrm{~kg}$. 750 à la fin du $4^{\mathrm{e}}$ mois), puis la baisse $\mathrm{s}^{\prime} a \mathrm{e}-$ centue et on arrive à $0 \mathrm{~kg} .640,0 \mathrm{~kg} .650,0 \mathrm{~kg}$. 600 , au $170^{\mathrm{e}}-180^{\mathrm{e}}$ jour et quelquefois même l'augmentation ne dépasse pas un demi-kilog. 
Dans lés premiers jours de leur existence, le mâle et la femelle accusent sensiblement la même augmentation pondérale moyenne ; mais, dès le $30^{\mathrm{e}}$ jour, l'égalité disparaît ; alors le poulain prend $1 \mathrm{~kg}$. 300 environ, la pouliche n'augmente que de $1 \mathrm{~kg}$. 250 , et cette différence s'accentue avec l'âge ; au $60^{\mathrm{e}}-70^{\mathrm{e}}$ jour, le poulain prend encore $1 \mathrm{~kg}$. et la pouliche $900 \mathrm{gr}$. seulement; à la fin du $4^{\mathrm{e}}$ mois, on note une augmentation de $700 \mathrm{gr}$. chez le mâle, et de 500-600 gr. chez la femelle. On nous a signalé des pouliches chez lesquelles le taux de croissance était tombé, à la fin du $6^{\mathrm{e}}$ mois, à $200 \mathrm{gr}$, 150 et même 100 grammes.

On peut donc conclure que, tout au début de l'existence, il y a égalité dans l'augmentation de poids chez le mâle et chez la femelle ; puis, bientôt, a pparaît une différence en faveur du mâle, différence qui s'accentue chaque jour davantage jusqu'au sevrage.

Cheval de trait. - Cornevin a mentionné quelques observations se rapportantà la croissance du cheval de trait. Dans une $1^{\text {re }}$ observation, il indique le poids d'un poulain, fils d'un père de $625 \mathrm{~kg}$. et d'une mère de 505 kilogs. Dans le premier mois de son existence, ce poulain accuse une augmentation pondérale moyenne de $1 \mathrm{~kg} .350$; pour le $2^{\text {e }}$ mois, cette augmentation de poids passe par un maximum et atteint 1 kilog; tout comme pour notre cheval anglo-arabe précédent, le taux de eroissance diminue progressivement pour atteindre au moment du sevrage $0 \mathrm{~kg}$. 796.

Une $2^{\text {e }}$ observation intéresse une pouliche née de parents pesant respectivement : père, $625 \mathrm{~kg}$. ; mère, $650 \mathrm{~kg}$.

Pendant le $1^{\text {er }}$ mois de sa vie, cette pouliche prit 740 grammes par jour ; pendant le $2^{\mathrm{e}}$ mois, $1 \mathrm{~kg} .500$; pendant le $3^{\mathrm{e}}$ mois, $1 \mathrm{~kg}$. 300 ; puis la diminution s'accentua, et durant les derniers mois de la période d'allaitement, l'augmentation journalière n'était que de 714 grammes en moyenne. Nous constatons dono un maximum pendant le $2^{\mathrm{e}}$ mois.

Une $3^{\text {e }}$ observation est celle d'un poulain de race commune. Dans le $1^{\mathrm{er}}$ mois, ee poulain prend $1 \mathrm{~kg}$. 600 par jour; dans les $2^{\mathrm{e}}$ et $3^{\mathrm{e}}$ mois, $1 \mathrm{~kg}$. 500; puis ensuite, jusqu'au sevrage, Ja baisse s'accentue et, dès le $4^{\mathrm{e}}$ mois, l'augmentation n'atteint plus $1 \mathrm{~kg}$.

A l'Ezole Nationale Vétérinaire de Lyon, nous avons pu peser un poulain, fils d'une jument commune, depuis sa naissance jusqu'au sevrage.

Pendant le $1^{\mathrm{er}}$ mois, il a accusé une augmentation de $1 \mathrm{~kg}$. 200 en moyenne; cette augmentation s'est encore accrue pendant les $2^{\mathrm{e}}$ et $3^{\mathrm{e}}$ mois, jusqu'à $1 \mathrm{~kg}$. 650 ; puis la diminution a commencé, et au $6^{\mathrm{e}}$ mois, le taux de croissance n'était plus que de 675 grammes en moyenne. (PI. XIII.)

De tous ces exemples on peut tirer les conclusions suivantes : 10 Qu'il s'agisse d'un cheval fin ou d'un cheval de trait, l'aug- 
mentation pondérale journalière passe par un maximum pendant le $2^{\mathrm{e}}$ mois de l'existence, puis diminue insensiblement jusqu'au sevrage. Le maximum varie entre $1 \mathrm{~kg}$. 100 et $1 \mathrm{~kg}, 600$; on peut l'évaluer en moyenne à $1 \mathrm{~kg}$. 400. Le minimum, atteint à partir du $5^{\mathrm{e}}$ mois, ou quelquefois au $6^{\mathrm{e}}$ seulement, peut être évalué en moyenne à $0 \mathrm{~kg}$. 750 environ.

\section{PL. XIII.}

\section{GROISSANGE QUOTIDIENNE DU POULAIN}

(Poids journalier de la naissance au $46^{\circ}$ jour)

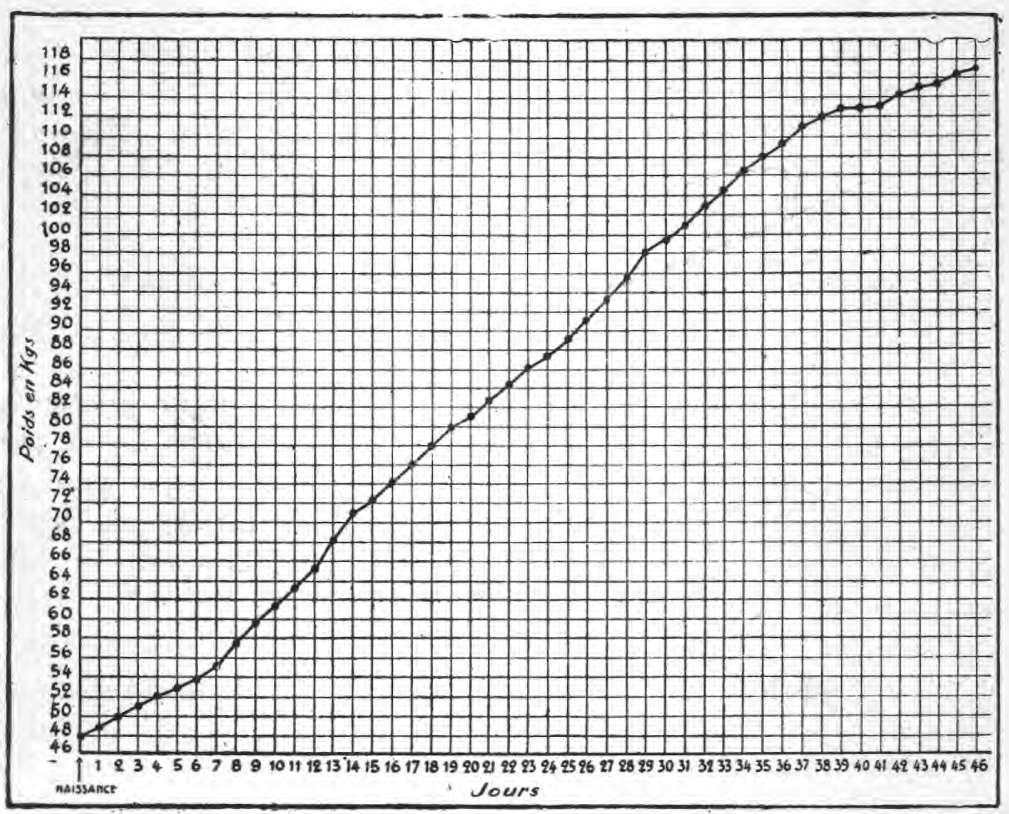

$2^{\circ}$ Les mâles croissent plus vite que les femelles. Au début de l'existence, le taux de croissance est à peu près semblable ; puis au $3^{\mathrm{e}}$ mois, très souvent dès le $2^{\mathrm{e}}$, les mâles accentuent leur accroissement, et cette différence se manifeste jusqu'au sevrage.

Voyons maintenant quelle est l'augmentation pondérale mensuelle pour des poulains de différentes races:

(a) Chevaux fins.

Race Arabe. Moyenne de la croissance mensuelle :

\begin{tabular}{|c|c|c|c|c|}
\hline $1^{\text {er mois }}$ & Kgs 35 & $4^{e} \mathrm{r}$ & ois & Kgs 18,500 \\
\hline $2^{\mathrm{e}}$ & $» 30$ & & p & Kgs 18 \\
\hline $3^{e} \quad n$ & 26,500 & $6^{\mathrm{e}}$ & " & 18 \\
\hline
\end{tabular}

Race Anglo.Arabe. Moyennes de la croissance mensuelle :

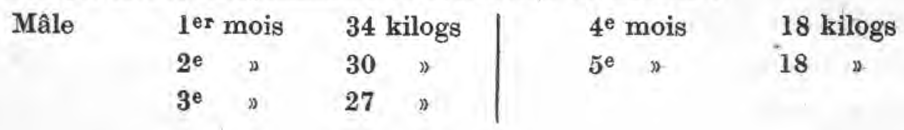




\begin{tabular}{|c|c|c|c|c|}
\hline Femelle & 1er mois & 34 kilogs & $4^{\mathrm{e}}$ mois & 22 kilogs \\
\hline . & $2^{\mathrm{e}}$ & $30 "$ & $5^{\mathrm{e}} \gg$ & 15 D \\
\hline & $3^{\mathrm{e}}$ & 28 " & $6^{\mathrm{e}}$ & 15 \\
\hline
\end{tabular}

L'accroissement mensuel est à peu près uniforme, quels que soient la race et le sexe; on peut l'évaluer en moyenne, d'une façon très générale, pour le cheval fin à :

\begin{tabular}{|c|c|c|c|}
\hline $1^{\mathrm{er}}$ mois & Kgs 35 & $4^{\mathrm{e}}$ mois & Kgs 19,500 \\
\hline $2^{e}$ & 30 & $5^{e}$ & 17 \\
\hline $3^{e}$ & 27 & $6^{\mathrm{e}}$ & 15 \\
\hline
\end{tabular}

Ces chiffres montrent combien l'accroissement diminue d'une façon régulière depuis la naissance jusqu'au sevrage ; le dernier mois de l'allaitement, le jeune n'augmente pas de la moitié par rapport au premier mois :

(b) Chevaux de trait.

$\begin{array}{lccc}1^{\text {er }} \text { mois } & \text { Kgs } 40 & \text { 40 mois } & \text { Kgs } 40 \\ 2^{\text {e }} & " 47,500 & 5^{\mathrm{e}} " & " 30 \\ 3^{\mathrm{e}} " & \# 45 & 6^{\mathrm{e}} " & " 30\end{array}$

Pendant un allaitement de 6 mois, le poids des poulains augmente en moyenne dans le rapport de 100 à 448 ; l'augmentation est plus considérable pour les chevaux de trait ( 5,22 environ) que pour les chevaux fins (mâle, 4,18 ; femelle, 4,04). L'augmentation journalière est en moyenne de $0 \mathrm{~kg}$. 900 pour chaque individu allaité (elle est plus forte pour les chevaux de trait que pour les autres).

Boussingault pense que l'on peut sans inconvénient sevrer à 3 mois ; à cet âge, le poulain a presque triplé son poids de naissance et a pris en moyenne $1 \mathrm{~kg} .04$ par jour.

Le poulain double son poids de naissance en 45 jours environ; il le triple entre 90 et 100 jours.

\section{B. - GROISSANGE PONDÉRALE DE L'ENFANT}

On a constaté que l'enfant accuse une diminution de poids dans les premiers jours qui suivent sa naissance. D'a près BouchaUd, cette perte serait de 65 gr. le premier jour, de $35 \mathrm{gr}$. le second.

En réalité, elle est beaucoup plus considérable ; elle atteint, en deux ou trois jours, 150 ou 200 gr. environ. Cette perte initiale est due à l'évacuation du méconium et de l'urine, et aussi, d'a près VARIOT, au fait que la montée du lait tardant à se faire, la ration de l'enfant est insuffisante.

La perte est plus considérable le premier jour (100 à 120 grammes) que les jours suivants. Certains enfants ne subissent pas cette perte de poids, ceux en particulier qui ont expulsé a vant l'accouchement le méconium et l'urine.

Dès le $4^{\mathrm{e}}$ jour, le jeune a repris son poids de naissance et la croissance se manifeste dès lors d'une façon continue. 
L'augmentation mensuelle de poids est, durant les quatre premiers mois, de 750 grammes en moyenne, soit 25 gr. par jour (J. Comby, Weill et Mouriquand, Nobécourt et Schreiber),

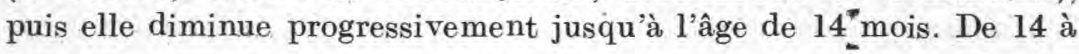

PL. XIV.

COURBE DU POIDS DE L'ENFANT PENDANT LES 10 PREMIERS JOURS (d'après P. Nobécourt)

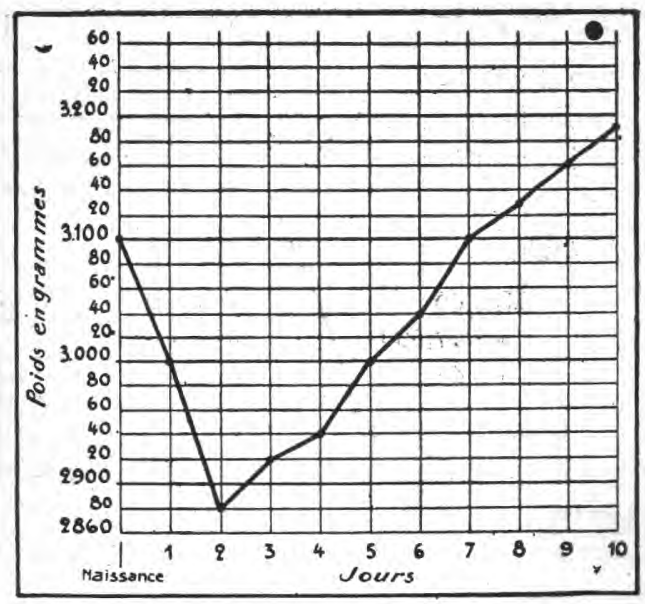

18 mois (âge de sevrage), l'augmentation mensuelle reste stationnaire et fixée à 200 grammes, ce qui correspond à un accroissement quotidien de 6 gr. 5 .

LESNÉ et Léon BINET indiquent comme augmentation journalière pendant la première année, chez un enfant normal et bien nourri :

25 à 30 gr. durant les quatre premiers mois.

15 à 20 "pendant les quatre mois suivants.

10 à 15 pendant les quatre derniers mois.

En résumé, l'enfant double son poids en quatre mois, quatre mois et demi ; autrement dit, un enfant die $3 \mathrm{~kg}$. à la naissance pèse, à 5 mois, $6 \mathrm{~kg}$. Il le triple en douze mois, ce qui fait en 7 mois une augmentation de 3 kilogs. L'enfant met done 8 mois à acquérir, dans la $2^{2}$ partie de l'année, ce qu'il avait mis 4 mois à gagner dans la première.

Les gains mensuels, au lieu de représenter une constante, diminuent d'une quantité qui varie de 50 à 100 grammes. Le gain quotidien est de 20 gr. pendant les 5 ou 6 premiers mois; de $15 \mathrm{gr}$. le $7^{\mathrm{e}}$ et le $8^{\mathrm{e}}$ mois ; d'environ $10 \mathrm{gr}$. pour les 3 derniers mois de l'année; à partir d'un an, la croissance est lente. La $2^{\mathrm{e}}$ année ne donne qu'une augmentation totale de $2 \mathrm{~kg}$. et demi à $3 \mathrm{~kg}$.

Nobécourt et Schreiber évaluent comme suit l'accroissement : 


De 0 à 4 mois
4 à 8 "
8 à $12 \quad$
12 à 14 "

Accroiss ement mensuel

$750 \mathrm{gr}$.
500 "
250 "
$250 "$

Accroissement quotidien

$$
\begin{array}{r}
25 \mathrm{gr} . \\
16 \\
8 ” \\
8 ”
\end{array}
$$

D'après Léon Binet et Lesné, pendant la seconde année, l'augmentation journalière de poids est de 8 grammes en moyenne pour

PL. XV.

GROISSANCE MENSUELLE DE L'ENFANT DE LA NAISSANCE A L'AGE DE 2 ANS

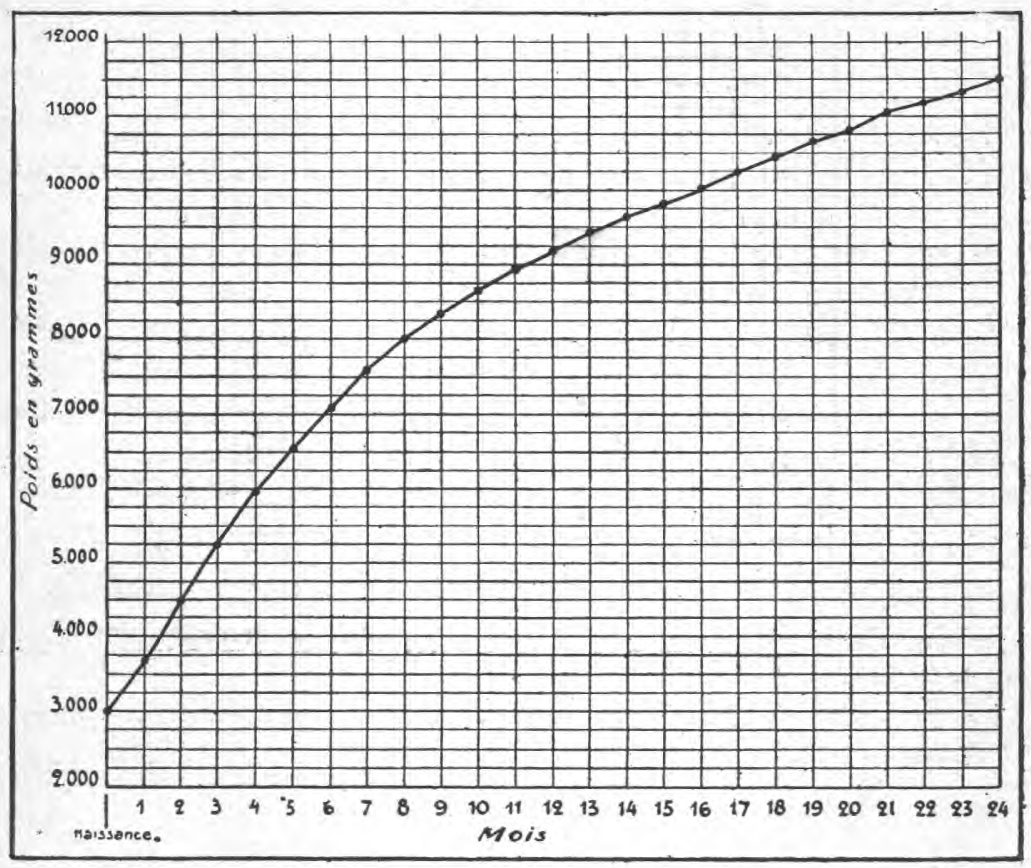

les premiers mois (250 gr. par mois) et de $5 \mathrm{gr}$. pour les derniers (150 gr. par mois). A deux ans, l'enfant a quadruplé son poids de naissance. Le poids des filles est légèrement inférieur à celui des garçons.

(A suivre.)

\section{REVUE}

\section{LA CONSTITUTION PHYSICO-CHIMIQUE DE LA POUDRE DE LAIT DESSÉCHÉ}

LES MATIẼRES GRASSES DE LA POUdRE DE LAIT DESSÉCHÉ PAR PULVÉRISATION

$$
\text { par G. GENIN }
$$

L'étude de la littérature scientifique montre qu'il a été fait de 